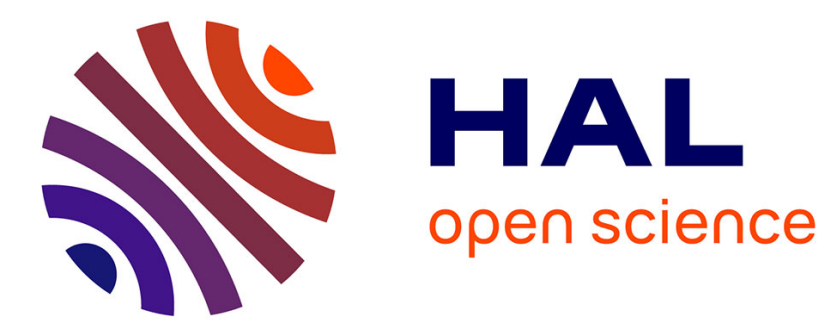

\title{
Breaking of non-Newtonian character in flows through a porous medium
}

Thibaud Chevalier, Stéphane Rodts, Xavier Chateau, Christophe Chevalier, Philippe Coussot

\section{- To cite this version:}

Thibaud Chevalier, Stéphane Rodts, Xavier Chateau, Christophe Chevalier, Philippe Coussot. Breaking of non-Newtonian character in flows through a porous medium. Physical Review E: Statistical, Nonlinear, and Soft Matter Physics, 2014, 89, pp.023002. 10.1103/physRevE.89.023002 . hal00944452

\section{HAL Id: hal-00944452}

https://hal-enpc.archives-ouvertes.fr/hal-00944452

Submitted on 10 Feb 2014

HAL is a multi-disciplinary open access archive for the deposit and dissemination of scientific research documents, whether they are published or not. The documents may come from teaching and research institutions in France or abroad, or from public or private research centers.
L'archive ouverte pluridisciplinaire HAL, est destinée au dépôt et à la diffusion de documents scientifiques de niveau recherche, publiés ou non, émanant des établissements d'enseignement et de recherche français ou étrangers, des laboratoires publics ou privés. 


\title{
Breaking of non-Newtonian character in flows through a porous medium
}

\author{
T. Chevalier, ${ }^{1}$ S. Rodts, ${ }^{1}$ X. Chateau, ${ }^{1}$ C. Chevalier, ${ }^{2}$ and P. Coussot ${ }^{1}$ \\ ${ }^{1}$ Laboratoire Navier (ENPC-IFSTTAR-CNRS), Université Paris-Est, Champs sur Marne \\ ${ }^{2}$ IFSTTAR, Université Paris-Est, Champs sur Marne \\ (Received 7 September 2013; published 6 February 2014)
}

\begin{abstract}
From NMR measurements we show that the velocity field of a yield stress fluid flowing through a disordered well-connected porous medium is very close to that for a Newtonian fluid. In particular, it is shown that no arrested regions exist even at very low velocities, for which the solid regime is expected to be dominant. This suggests that these results obtained for strongly nonlinear fluid can be extrapolated to any nonlinear fluid. We deduce a generalized form of Darcy's law for such materials and provide insight into the physical origin of the coefficients involved in this expression, which are shown to be moments of the second invariant of the strain rate tensor.
\end{abstract}

DOI: 10.1103/PhysRevE.89.023002

\section{INTRODUCTION}

Flows of non-Newtonian fluids through porous media are involved in a wide range of applications [1], such as the injection of cement in soils, penetration of glue in porous substrates, propagation of blood through the kidneys, hydraulic fracture [2], etc. One of the most important applications concerns oil reservoir engineering, in which a wide range of complex fluids (foams, polymers, emulsions) are used to improve oil recovery [3]. In these processes the flow characteristics of such fluids in porous media remain poorly known due to the complexity of the fluid behavior and the opacity of the solid structure.

For Newtonian fluids (of constant viscosity $\mu$ ) we know that any element of the liquid network flows, and in the absence of inertia effects, the velocity field scaled by the average velocity $(\bar{V})$ is constant (Stokes flow). This is at the origin of Darcy's law, which tells us that the pressure gradient $(\nabla p)$ is proportional to $\bar{V}$ by a factor $\mu / K$, in which $K$ is a characteristic of the porous medium, namely, its permeability. For nonlinear fluids we expect a significant evolution of the distribution of flowing regions with the velocity and thus a more complex relationship between $\nabla p$ and $\bar{V}$.

For yield stress fluids (mud, cement, concentrated emulsions, or foams), which flow like liquids only beyond a critical stress and behave as solids otherwise, various analytical or numerical approaches [4] have been developed but the validity of their physical assumptions could not be checked. Some in-depth physical studies suggested that two critical effects could occur: (i) at the pore scale the flowing volume increases with the pressure gradient [5] and (ii) at a macroscopic scale the flow starts as a percolation effect, i.e., at a critical pressure drop liquid regions exist only along a specific path throughout the porous medium [5,6], and as $\nabla p$ is increased more flowing paths progressively form within the porous medium. Such effects still need experimental validation.

Here we observe a yield stress fluid flow inside a model bead packing with a NMR technique which provides straightforward information on the average local flow characteristics without being affected by any spatial resolution problem. We show that there is no region of fluid at rest, even at low $\bar{V}$ values, and the velocity density distribution is similar to that of a Newtonian fluid. This makes it possible to deduce the effective form of Darcy's law for yield stress fluids and the physical origin of its coefficients.
PACS number(s): 47.56.+r, 47.50.-d, 83.60.La

\section{MATERIALS AND PROCEDURES}

Our yield stress fluid is a water-in-oil emulsion $(81 \%$ water, $19 \%$ oil $)$ prepared by dispersing a water $+\mathrm{CaCl}_{2}(150 \mathrm{~g} / \mathrm{L})$ salt solution in a surfactant solution (dodecane $+7.5 \mathrm{wt} \%$ Span 80 surfactant) with a mixer. The droplet size ranges from 1 to $5 \mu \mathrm{m}$. Rheological tests in simple shear show that this is a simple yield stress fluid (with negligible thixotropic character) with a flow curve that can be well represented by a Herschel-Bulkley (HB) model (see Fig. 1): $\tau<\tau_{c} \Rightarrow \dot{\gamma}=0$ (solid regime); $\tau>\tau_{c} \Rightarrow \tau=\tau_{c}+k \dot{\gamma}^{n}$ (liquid regime), in which $\tau$ is the shear stress, $\dot{\gamma}$ the shear rate, $\tau_{c}$ the yield stress, and $k$ and $n$ two material parameters [7]. The fluid is pushed by a piston through a duct $(8 \mathrm{~cm}$ diameter) and then penetrates a bead packing. Two types of beads were used with a similar (when represented as a function of the size rescaled by the mean diameter) triangular grain size distribution in the range $0.8-1.2$ times the average diameter $D=0.5$ or $2 \mathrm{~mm}$. They were covered with a thin layer $(\approx 0.015 D)$ of resin which stuck them together, leading to a porosity $\varepsilon \approx 0.33$.

The generalized Reynolds number, i.e., $\operatorname{Re}=\rho \bar{V}^{2} / \tau_{c}$, in which $\rho$ is the fluid density, is much smaller than 1 , which means inertia effects are negligible. Under these conditions the flow is governed by the Bingham number, $\mathrm{Bi}=\tau_{c} / k \dot{\gamma}^{n}$, which estimates the ratio of the constant (elastoplastic) to the rate-dependent (viscous) parts of the constitutive equation, and finally gives an idea of the relative importance of the solid and liquid regions in the sample. Thus flows through similar porous media with different pore sizes or yield stress values have similar flow characteristics (after appropriate rescaling) as soon as they have the same Bingham number.

For the flow through a complex geometry we use a characteristic shear rate $(\dot{\gamma}=V / d)$, which is the ratio of the average velocity through the void volume $(V)$ to a characteristic length scale of the cross section of the geometry $(d)$. For our bead packing we have $V=\bar{V} / \varepsilon$ and we take $d=D / 6$. Our range of velocities ( $\bar{V}$ from 0.013 to $\left.1.3 \mathrm{~mm} \mathrm{~s}^{-1}\right)$ and bead sizes lead to Bi ranging from 1 to 11.4 , which includes the main classes of yield stress fluid flows, i.e., (i) almost purely plastic flows $(\mathrm{Bi} \gg 1)$ and (ii) flows with both elastoplastic and viscous effects playing a significant role $(\mathrm{Bi} \approx 1)$.

Measurements were carried out after a time of flow (after saturation of the bead packing) such that the distance (typically $10 \mathrm{~cm}$ ) covered at the average velocity was several orders of magnitude larger than the bead size. This ascertains that a 


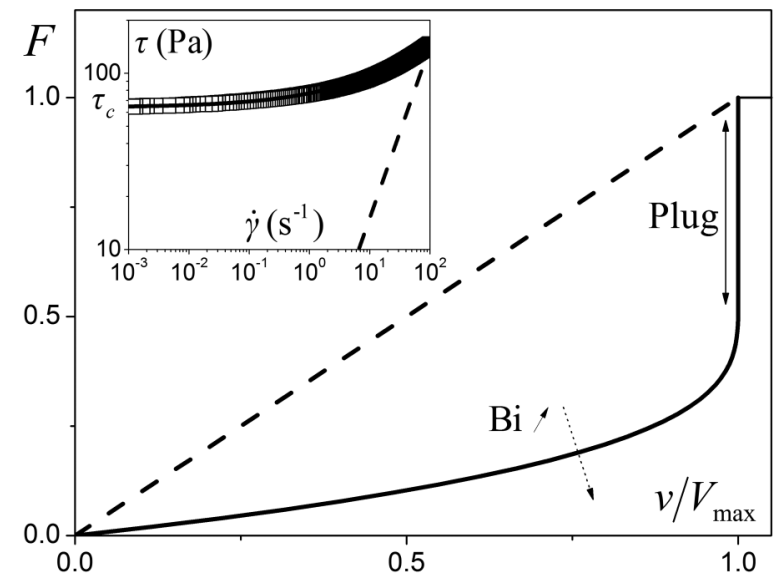

FIG. 1. Distribution function vs velocity scaled by the maximum velocity for flows through a capillary: Newtonian fluid (dashed line) and yield stress fluid (continuous line) following a HB model with an unsheared region (plug) extending over $2 / 3$ of the capillary radius (i.e., $\mathrm{Bi}=5.4$ ). Inset: experimental flow curve of our material (squares) fitted by a HB model [7] (continuous line), and Newtonian flow curve of arbitrary viscosity (dashed line).

steady-state flow was reached and residual creep motions in the solid regions were negligible.

The whole setup is inserted vertically in the channel of a vertical proton magnetic resonance imaging (MRI) system (Avance 24/80 DBX by Bruker, 0.5-T superconducting magnet by Oxford, $20-\mathrm{cm}$ inner diameter). Velocity distributions in the pore space are measured by means of pulsed-gradient spin echo (PGSE) proton NMR [8] as follows: After an initial excitation of the hydrogen spin system of liquid molecules, a pair of self-compensating field gradient pulses of value $\vec{G}$ produces in the spin precession a motion-sensitive phase shift $\delta \varphi=\gamma \vec{G} \cdot\left(\int_{0}^{\delta} \vec{r}(t) d t-\int_{\Delta}^{\Delta+\delta} \vec{r}(t) d t\right)$, where $\gamma$ is the gyromagnetic ratio, $\vec{r}(t)$ the molecular trajectory, and time constants $\delta$ and $\Delta$ define the timing of the pulse sequence. The NMR protocol uses various gradient intensities to recover statistical information on the bracketed term [9]. The latter corresponds to local velocity field components by some known multiplicative factor, provided molecular self-diffusive motions can be neglected and sequence timing is short. The sequence was designed here to be sensitive only to water phase. Water confinement in micrometer-size droplets strongly restricts diffusive motions. Residual diffusion inside droplets was quantified [10] so as to find sequence tunings which kept the spurious effects of water diffusion on velocity distributions under some undetectable level [11]. Results are presented in terms of a probability density function of one velocity component (also often called "1D velocity propagator"), which is such that the fraction of fluid having a velocity between $v$ and $v+d v$ is $f(v) d v$. We checked the ability of the technique to measure the effective velocity distribution for flows in simple geometry, including arrested regions (see, for example, Fig. 2)

In order to appreciate the data in a straightforward way, we think it more appropriate to look at the distribution function, defined as $F(v)=\int_{-\infty}^{v} f(u) d u$. Indeed, the shape of $F$ is less dependent on the measurement uncertainties, in particular,

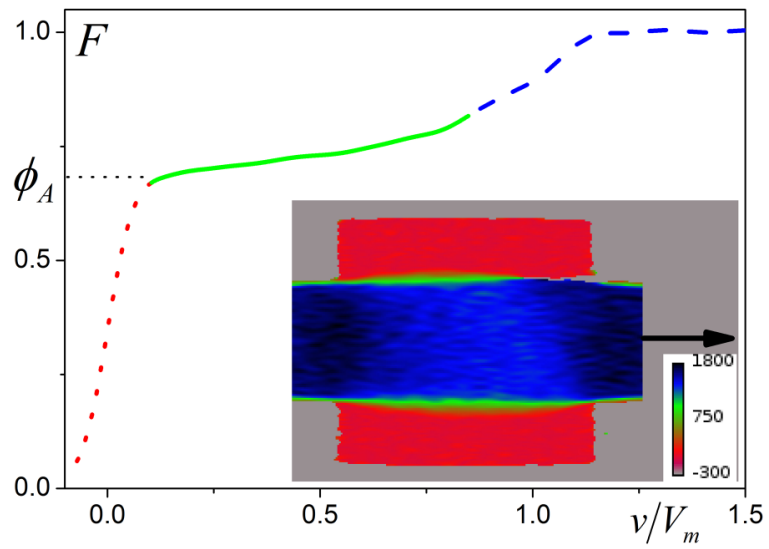

FIG. 2. (Color online) Distribution function vs velocity scaled by the mean velocity (in the small duct) measured for the flow of a yield stress fluid through a 7-cm-diameter and 7-cm-long duct between 3.5$\mathrm{cm}$-diameter ducts for $\mathrm{Bi}=14$. The colored regions approximately correspond to those which are visible on the velocity field (see inset) obtained from independent measurements (see [13]) and represented by color scale (in $\mathrm{mm} / \mathrm{s}$ ): outer arrested region (red and gray dotted lines), transitional region (light green, continuous line), moving plug region (dark blue, dashed line).

the impact of the noise is damped as the integral filters out some noise components [11]. In the following, except when mentioned, we will focus on the longitudinal velocity, i.e., along the cylindrical sample axis. For example, for the flow through a capillary, $F$ takes very different shapes depending on the rheological behavior of the fluid (see Fig. 1), which means that the distribution function can be a fingerprint of this behavior. For a yield stress fluid there is an unsheared region (plug) around the center of the duct which is associated with a vertical jump at the end of the distribution (see Fig. 1). The size of this region increases with $\mathrm{Bi}$. On the contrary, if the fluid is arrested in a region of volume fraction $\phi_{A}$, such as in an expansion-contraction, the distribution globally starts by a jump to a finite level $\left[F(0)=\phi_{A}\right]$ (see Fig. 2).

\section{EXPERIMENTAL DATA AND ANALYSIS}

The first critical result from our data for the flow through a bead packing is that over our whole range of $\mathrm{Bi}$ the distribution function starts around zero, i.e., $F(0) \approx 0.05$ (see Fig. 3). More precisely, in contrast with the distribution for the model pore of Fig. 2, the overall shape (i.e., leaving apart possible uncertainties around zero) of the curves for porous media does not exhibit any apparent step around zero which might be associated with a more significant arrested region. Similar conclusions can be drawn from transversal distributions (see inset of Fig. 3). For a yield stress fluid in steady flow this means that the whole fluid is in the liquid regime and flows, i.e., the arrested (solid) regions occupy a negligible volume. This observation is in contradiction with previous conceptual expectations for such flows [5,6]. In particular, our result implies that even at low velocity there is no preferential pathway involving a fraction of the pores through which the fluid would flow while it would be arrested outside. Our data also prove that at the scale of the pore there is no significant 


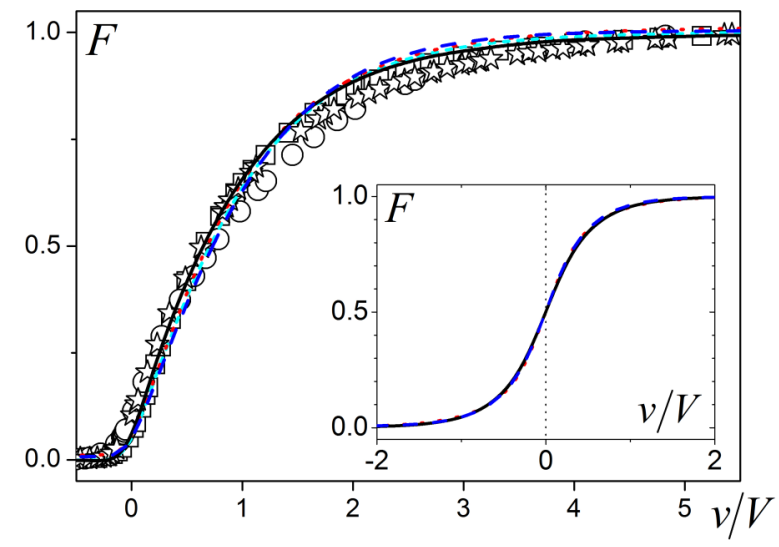

FIG. 3. (Color online) Distribution functions as a function of the velocity scaled by the average velocity through the voids. (i) Computed from literature NMR data for $f$ (at sufficiently small $\Delta$ ) with Newtonian fluids (water or oil) through bead packings: (squares) Fig. 1 of [15] $V=3.55 \mathrm{~mm} \mathrm{~s}^{-1}$; (circles) data from Fig. 4 of [16] $V=1.1 \mathrm{~mm} \mathrm{~s}^{-1}$, and (stars) for a power-law fluid (Xanthan), Fig. 9 of [17], $V=30 \mathrm{~mm} \mathrm{~s}^{-1}$. (ii) From our tests with a yield stress fluid: $(D=2 \mathrm{~mm})$ short dash light blue $\left(V=0.12 \mathrm{~mm} \mathrm{~s}^{-1}, \mathrm{Bi}=7.3\right)$ and dotted red line $\left(V=4 \mathrm{~mm} \mathrm{~s}^{-1}, \mathrm{Bi}=1.8\right) ;(D=0.5 \mathrm{~mm})$ black line $\left(V=0.12 \mathrm{~mm} \mathrm{~s}^{-1}, \mathrm{Bi}=4.2\right)$ and dashed dark blue $\left(V=4 \mathrm{~mm} \mathrm{~s}^{-1}\right.$, $\mathrm{Bi}=1.04)$. Our data for intermediate velocities fall exactly along the other data. The data for $\mathrm{Bi}=11.4$ are similar although slightly more affected by diffusion. The inset shows the distribution function for the transversal velocity for $D=0.5 \mathrm{~mm}$, and $V=0.4 \mathrm{~mm} \mathrm{~s}^{-1}, \mathrm{Bi}=3$ (black), $V=1.2 \mathrm{~mm} \mathrm{~s}^{-1}, \mathrm{Bi}=1.7$ (dotted red), and $V=4 \mathrm{~mm} \mathrm{~s}^{-1}$, $\mathrm{Bi}=1.04$ (dashed dark blue).

volume of arrested fluid; otherwise this would occur in a large number of pores, leading, on average, to a significant fraction of arrested fluid.

This is a surprising result, since in a model pore made of a short duct between two smaller ducts and for a similar range of $\mathrm{Bi}$ there remains, around the central flowing region, a wide region of arrested fluid where the stress is necessarily smaller than the yield stress [12,13] (see Fig. 2). Moreover, in order to induce the flow of a yield stress fluid between two long plates situated at a distance $h$, the minimum pressure gradient to apply is $2 \tau_{c} / h$, which tends to infinity when $h$ tends to zero. Thus in a bead packing we could expect a stoppage in the regions situated between two very close beads. We suggest that this does not occur, because such regions extend over short distance and are connected in various directions with other regions containing larger fluid volumes. Finally, the picture we get from these data is that instead of keeping some restricted regions at rest, a liquid regime sets up everywhere in the fluid network.

Let us now compare the distribution functions obtained for different porous media and at different $V$ by representing them as a function of the velocity scaled by $V$. Owing to small $\Delta$ and $\delta$ imposed by NMR sequence tuning, this is equivalent to looking at the distribution of local displacements scaled by the mean displacement. With such a representation, for flows of Newtonian fluids in the absence of inertia effects (i.e., low $\mathrm{Re}$ ), we expect a single distribution function for different $V$ or similar porous media of various length scales. For example, in a capillary, $F$ is a straight line in any case. On the contrary, for a yield stress fluid $F$ varies widely with the Bingham number, which depends on the velocity and capillary diameter (see Fig. 1).

Surprisingly, for a yield stress fluid through a bead packing, in our range of experimental conditions, the distribution function in the transversal and longitudinal directions are independent of $V$ and $D$ (see Fig. 3). Thus, once again in contrast with yield stress fluid flows through simple geometry, $V$ has no significant impact on the velocity distribution. Actually, such a phenomenon has already been observed for a complex flow (extrusion) of yield stress fluids [14] in a similar range of $\mathrm{Bi}$ : over a length of the entrance conduit approximately equal to its diameter, the velocity fields (in a longitudinal cross section) scaled by the piston velocity appear quite similar for different $\mathrm{Bi}$.

Since the independence of $F$ with regard to $V$ and $D$ is typically obtained for a Newtonian fluid, our results suggest that the local flow characteristics of a yield stress fluid through a bead packing are similar to those of a Newtonian fluid. This conclusion is further supported by the observation that the distribution function for the yield stress fluid is approximately similar to that for a Newtonian fluid (see Fig. 3) and to that for a simple shear-thinning fluid (without yield stress).

It could be argued that there is an effect of statistical averaging over the various local geometrical shapes leading to the same average distribution whatever the differences at a local scale. However, even after this transformation the final distribution should depend on the specific local characteristics of the fluid flow. Indeed, if we assume that in a given pore (i.e., a void region), due to a different rheological behavior we have a velocity distribution different from that for a Newtonian fluid, e.g., a larger fraction with high velocities, we will have a similar effect in another pore of different shape and finally a similar effect on the average over all pores, with some impact on $F$. Thus we can conclude that the local velocity fields for the different fluid types are very close; otherwise we would not get similar distribution functions.

\section{DISCUSSION}

These observations lead us to conclude that the strong differences of flow characteristics usually induced by different rheological behavior types, such as can be observed in a capillary (see Fig. 1), are "broken" by the porous medium. This conclusion is further supported by the observation that the yielding character of a fluid is broken around the entrance in a small die, which well represents the situation continuously encountered inside a bead packing: far from the entrance there is a plug flow [14], but just after the entrance the velocity profile is close to that for a Newtonian fluid and only after some distance of the order of the conduit diameter the standard profile including a plug is recovered (see Fig. 4). Finally, the corresponding distribution function in cross section evolves as described in Fig. 1, i.e., from that of a simple fluid to that of a yield stress fluid (see Fig. 4).

We consider that the process at the origin of the breakage of the non-Newtonian character in a bead packing is as follows: The fluid is continuously and significantly deformed in various directions as it moves through a complex porous network. As soon as it has been significantly deformed so as to reach a velocity field similar to that of a Newtonian fluid, it will never 


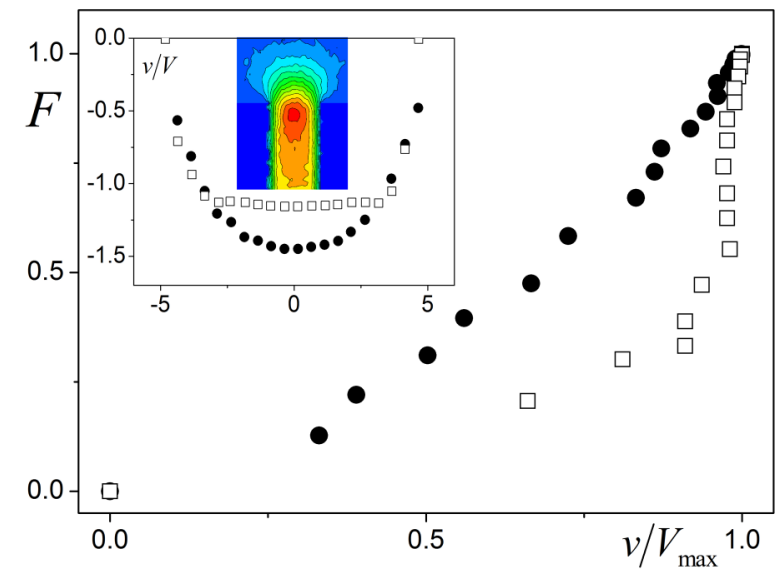

FIG. 4. (Color online) Yield stress fluid flow (arriving from a 8 -cm-diameter duct) through a die of $1 \mathrm{~cm}$ diameter. Distribution function in cross sections at $1 \mathrm{~mm}$ (circles) and $16 \mathrm{~mm}$ (squares) from the die entrance, calculated from the velocity profiles (see inset) (data from [14]). The velocity field in color scale [highest velocities in red (central region), smallest ones in blue (outside region)] is also shown in the inset.

encounter a region allowing it to recover its flow characteristics typical of a yield stress fluid in uniform flow, and thus will keep a Newtonian velocity field.

We can now use these results to establish a general form of Darcy's law for some complex fluids. In particular, for a yield stress fluid the usual three-dimensional form of the constitutive equation [14], which leads to a HB model in simple shear, is $\tau_{I I}<\tau_{c} \Rightarrow \mathbf{d}=0$ (solid regime) and $\tau_{I I}>\tau_{c} \Rightarrow \boldsymbol{\tau}=$ $\tau_{c} \mathbf{d} / d_{I I}+2^{n} k d_{I I}^{n-1} \mathbf{d}$ (liquid regime), in which $\boldsymbol{\tau}$ is the deviatoric stress tensor, $\mathbf{d}$ the strain rate tensor, and $d_{I I}=\sqrt{\operatorname{trd}^{2} / 2}$. The viscous dissipation inside the bead packing (of volume $\Omega$ ) is $P=\int_{\Omega} \operatorname{tr}(\mathbf{d} \cdot \boldsymbol{\tau}) d \omega$. Introducing the constitutive equation we deduce $P=\tau_{c} \int 2 d_{I I} d \omega+k \int\left(2 d_{I I}\right)^{n+1} d \omega$, which may be rewritten as $P=\alpha \tau_{c} \varepsilon \Omega(V / D)+\beta_{n} \varepsilon \Omega(V / D)^{n+1}$, with $\alpha=(1 / \varepsilon \Omega) \int 2 \frac{D}{V} d_{I I} d \omega, \beta_{n}=(1 / \varepsilon \Omega) \int\left(2 \frac{D}{V} d_{I I}\right)^{n+1} d \omega$.

The above experimental results concerning the approximately unique scaled velocity field obtained for different velocities and bead diameters lead to the conclusion that $(D / V) d_{I I}$ is a unique dimensionless function weakly dependent on the effective behavior of the material. That means that $\alpha$ and $\beta_{n}$ are two dimensionless coefficients depending only on this distribution of shear rate intensity and on the coefficient $n$. Since the viscous dissipation may also be expressed through a macroscopic approach as $\Omega \nabla p \cdot \bar{V}=\varepsilon \Omega \nabla p \cdot V$, we deduce Darcy's law for yield stress fluids (which includes power-law and Newtonian fluids):

$$
\nabla p=\alpha \frac{\tau_{c}}{D}+\beta_{n} k \frac{1}{D}\left(\frac{V}{D}\right)^{n}
$$

The form of this law is consistent with the analysis of a series of measurements of $\nabla p$ as a function of $V$ for different yield stress fluids and pore sizes [18]. Our approach now demonstrates that this is the general form of Darcy's law for yield stress fluids and provides explicit expressions and the physical meaning for the two coefficients $\left(\alpha\right.$ and $\beta_{n}$ ).

\section{CONCLUSION}

Finally, our experimental results suggest a change of paradigm for dealing with transport in porous mediums, i.e., the fact that the flow of a nonlinear fluid in a complex geometry tends to somehow cancel its rheological complexity. The complete origin of this effect still needs to be understood by theory or simulations, for example, via the promising Lattice-Boltzmann approaches $[19,20]$.
[1] J. G. Savins, Ind. Eng. Chem. 61, 18 (1969).

[2] A. T. Unwin, Math. Eng. Ind. 5, 59 (1994).

[3] J. R. A. Pearson and P. M. J. Tardy, J. Non-Newt. Fluid Mech. 102, 447 (2002).

[4] H. Pascal and F. Pascal, Int. J. Eng. Sci. 23, 571 (1985); T. Al-Fariss and K. L. Pinder, Can. J. Chem. Eng. 65, 391 (1987); G. C. Vradis and A. L. Protopapas, J. Hydr. Eng. 119, 95 (1993); R. K. Dash, K. N. Mehta, and G. Jayaraman, Int. J. Eng. Sci. 34, 1145 (1996); J. R. A. M. Chena, W. Rossenb, and Y. C. Yortsos, Chem. Eng. Sci. 60, 4183 (2005); G. G. Chase and P. Dachavijit, Rheol. Acta 44, 495 (2005); T. Sochi and M. J. Blunt, J. Pet. Sci. Eng. 60, 105 (2008).

[5] M. T. Balhoff and K. E. Thompson, AIChE J. 50, 3034 (2004).

[6] M. Sahimi, AIChE J. 39, 369 (1993).

[7] Here we have $\tau_{c}=63 \mathrm{~Pa}, k=13 \mathrm{~Pa} \mathrm{~s}^{n}$, and $n=0.4$.

[8] E. O. Stejskal and J. E. Tanner, J. Chem. Phys. 42, 288 (1965).

[9] P. Callaghan, Principles of Nuclear Magnetic Resonance Microscopy (Oxford University Press, Oxford, 1991).

[10] C. Neuman, J. Chem. Phys. 60, 4508 (1974).

[11] T. Chevalier, Ph.D. thesis, Univ. Paris-Est, France (in French) (2013), http://tel.archives-ouvertes.fr/tel-00903850
[12] P. R. de Souza Mendes, M. F. Naccache, P. R. Varges, and F. H. Marchesini, J. Non-Newt. Fluid Mech. 142, 207 (2007).

[13] T. Chevalier, S. Rodts, X. Chateau, J. Boujlel, M. Maillard, and P. Coussot, Europhys. Lett. 102, 48002 (2013).

[14] B. D. Rabideau, P. Moucheront, F. Bertrand, S. Rodts, N. Roussel, C. Lanos, and P. Coussot, J. Non-Newt. Fluid Mech. 165, 394 (2010).

[15] L. Lebon, J. Leblond, J. P. Hulin, N. S. Martys, and L. M. Schwartz, Magn. Reson. Imaging 14, 989 (1996).

[16] J. J. Teyssier, K. J. Packer, J. F. Thovert, and P. M. Adler, AIChE J. 43, 1653 (1997).

[17] D. Mertens, C. Heinen, E. H. Hardy, and H. W. Buggisch, Chem. Eng. Technol. 29, 854 (2006).

[18] T. Chevalier, C. Chevalier, X. Clain, J. C. Dupla, J. Canou, S. Rodts, and P. Coussot, J. Non-Newt. Fluid Mech. 195, 57 (2013).

[19] E. Aharonov and D. H. Rothman, Geophys. Res. Lett. 20, 679 (1993).

[20] A. Vikhansky, J. Non-Newt. Fluid Mech. 155, 95 (2008); L. Talon and D. Bauer, Eur. Phys. J. E 36, 139 (2013). 